\title{
INFLUENCE DE LA TEMPÉRATURE SUR LE DÉVELOPPEMENT DE LA PUCE AFRICAINE DU CHAT CTENOCEPHALIDES FELIS STRONGYLUS (JORDAN, 1925) (SIPHONAPTERA : PUlICIDAE)
}

\author{
YAO K.P.*, N'GORAN K.E.* \& FRANC M.**
}

Summary : INFLUENCE OF THE TEMPERATURE ON THE DEVELOPMENT OF THE AFRICAN CAT FLEA CTENOCEPHALIDES FELIS STRONGYLUS (JORDAN, 1925) (SipHONAPTERA : Pulicidae)

Ctenocephalides felis (Bouché, 1835) commonly called "cat's flea" presents two recognized subspecies: Ctenocephalides felis strongylus (Jordan, 1925), observed in the African continent, and Ctenocephalides felis felis (Bouché, 1835) in the other regions (North Africa, Europe and America) (Ménier and Beaucournu, 1999). In sub-Saharan Africa, the principal flea found in the pets and certain livestock (ovine, caprine and bovine), belongs to the subspecies C. f. strongylus. Some bio-ecologic parameters of C. f. strongylus were studied in various conditions of breeding and the results compared with those currently available for $C$. f. felis. At $75 \% \pm 5$ of relative humidity, the development cycle of C. f. strongylus lasts $20-21$ days at $27^{\circ} \mathrm{C}$ and 16 to 17 days at $29^{\circ} \mathrm{C}$. In comparison with C. f. felis, it is shown that for identical breeding temperatures, the African sub-species of the cat flea develops itself slowly. This difference could be explained by the influence of the climate of their respective areas of distribution on their development cycle. With $75 \% \pm 5$ of relative humidity, C. f. strongylus cannot survive more than 14 days in temperatures ranging between 27 and $29^{\circ} \mathrm{C}$, and this without any blood meal. Under the same conditions, this duration of survival does not exceed 16 days at $19^{\circ} \mathrm{C}$. But when C. f. strongylus has taken a first blood meal, its lifespan is much shorter when it is out of its host. Indeed, no individual is found living three days passed out of the fur of its host at $29^{\circ} \mathrm{C}$, five days at $27^{\circ} \mathrm{C}$ and eight days at $19^{\circ} \mathrm{C}$. It is the same for $C$. f. felis. These data on bio-ecology of C. f. strongylus enable to understand the influence of temperature on its

development cycle and consider more efficient strategies of control.

KEY WORDS : Ctenocephalides felis strongylus, Ctenocephalides felis felis, flea, development cycle, temperature.

\footnotetext{
I
}

es facteurs écologiques tels que la température et l'humidité ont une influence majeure sur le développement des êtres vivants, en particulier sur certains insectes. En Afrique, la puce du chat (Ctenocephalides felis (Bouché, 1835)) signalée par Curasson

\footnotetext{
* UFR Biosciences, Université de Cocody-Abidjan, 22 BP 582, Abidjan 22, Côte d'Ivoire.

** UMR 181, École Nationale Vétérinaire de Toulouse, 23, Chemin des Capelles, 31076 Toulouse Cedex, France.

Correspondance : Kouassi Patrick Yao

22 BP 518, Abidjan 22, Côte d'Ivoire.

Tél. : +225-04-296-250

E-mail : patrick.yao@univ-cocody.ci
}

\section{Résumé :}

Ctenocephalides felis (Bouché, 1835) communément appelée "puce du chat" présente deux sous-espèces reconnues Ctenocephalides felis strongylus Uordan, 1925) inféodée au continent africain et Ctenocephalides felis felis (Bouché, 1835) présente dans les zones à climat tempéré (Afrique du Nord, Europe et Amérique) (Ménier et Beaucournu, 1999). En Afrique subsaharienne, la principale puce retrouvée chez les animaux de compagnie et chez certains animaux d'élevage lovins, caprins et bovins) appartient à la sous-espèce C. f. strongylus. Quelques paramètres bio-écologiques de C. f. strongylus ont été étudiés dans différentes conditions d'élevage. Les résultats ont été comparés à ceux de C. f. felis actuellement disponibles. À $75 \% \pm 5$ d'humidité relative, le cycle de développement de C. f. strongylus dure 20-21 jours à $27^{\circ} \mathrm{C}$ et de 16 à 17 jours à $29^{\circ} \mathrm{C}$. Ainsi, la sousespèce africaine de la puce du chat (C. f. strongylus) se développe moins vite que C. f. felis à températures identiques. Cette différence pourrait s'expliquer par l'influence du climat de leurs aires de distribution respectives sur leur cycle de développement. À $75 \%$ \pm 5 d'humidité relative, les adultes de C. f. strongylus ne peuvent survivre plus de 14 jours dans l'environnement à des températures comprises entre 27 et $29^{\circ} \mathrm{C}$, lorsqu'elles n'ont jamais pris de repas sanguin. Dans ces mêmes conditions, la durée de survie n'excède pas 16 jours à $19^{\circ} \mathrm{C}$. Mais lorsque C. f. strongylus a pris un premier repas de sang, elle a une durée de vie beaucoup plus courte lorsqu'elle est hors de son hôte. En effet, aucun individu n'est retrouvé vivant trois jours passé hors de la fourrure de son hôte à $29^{\circ} \mathrm{C}$, cinq jours à $27^{\circ} \mathrm{C}$ et huit jours à $19^{\circ} \mathrm{C}$. II en est de même pour C. f. felis. Ces données sur la bio-écologie de C. f. strongylus permettent de comprendre l'influence de la température sur son cycle de développement et d'envisager des stratégies de lutte plus efficientes.

MOTS CLÉS : Ctenocephalides felis strongylus, Ctenocephalides felis felis, puce, cycle de développement, température.

(1925) et Opasina (1983) appartient à la sous-espèce Ctenocephalides felis strongylus (Jordan, 1925) (Ménier et Beaucournu, 1999). En 2002, la puce incriminée lors des infestations massives des ovins dans le centre de la Côte d'Ivoire appartenait à la sous-espèce C.f. strongylus (Ouattara, comm. pers.) ; il en a été de même en Lybie (Kaal et al., 2006). Outre ces observations, C.f. strongylus a été signalée comme étant la principale puce observée chez les animaux de compagnie (chiens et chats) en Côte d'Ivoire ; de plus elle a été citée comme deuxième ectoparasite d'importance médico-vétérinaire, après la tique (Yao, 2007). 
Dans le cadre d'une lutte efficace contre les puces, il apparaît important de s'appuyer sur la compréhension de sa biologie, de ses hôtes et de leur environnement commun. Dans cet environnement, la température et l'humidité relative représentent deux composantes essentielles. Le présent travail vise principalement à relever l'influence de la température sur le cycle de développement de C.f. strongylus et la survie des adultes dans l'environnement, après la prise d'un repas de sang ou non.

\section{MATÉRIEL ET MÉTHODES}

I a sous-espèce africaine de la puce du chat (C.f. strongylus) représente le matériel biologique des chats de race européenne. Nos résultats sont comparés à ceux obtenus par différents auteurs ayant utilisé la deuxième sous-espèce, en l'occurrence C.f. felis (Bouché, 1835). Les individus de C.f. strongylus utilisés proviennent de chiens infestés examinés à Abidjan. Ces puces ont été mises en élevage à l'UMR 181 de l'École Nationale Vétérinaire de Toulouse (France).

\section{CyCle De DÉVELOPPEMENT}

Dans deux étuves, l'une portée à $29^{\circ} \mathrm{C}$ et l'autre à $27^{\circ} \mathrm{C}$ avec une humidité relative de $75 \% \pm 5,1500$ œufs de C.f. strongylus répartis en trois lots de 20 pots cylindriques, sont mis en incubation dans chaque étuve. Les pots, contenant chacun 25 oufs avec un milieu nutritif, sont recouverts de papier filtre et vissé par un couvercle percé.
Chaque jour pour la température de $29^{\circ} \mathrm{C}$ et tous les deux jours pour celle de $27^{\circ} \mathrm{C}$, un pot par lot (répétition) est retiré des étuves afin d'en examiner le contenu. L'examen du milieu nutritif est réalisé sous loupe binoculaire. Les individus de chaque stade de développement (L1, L2, L3, cocon et imago) sont identifiés puis dénombrés.

\section{SURVIE DE C. F. STRONGYLUS \\ À DIFFÉRENTES TEMPÉRATURES}

L'humidité relative est maintenue constante durant tout l'essai, soit $75 \% \pm 5$ et trois étuves sont utilisées aux températures suivantes : $19^{\circ} \mathrm{C}, 27^{\circ} \mathrm{C}$ et $29^{\circ} \mathrm{C}$. Deux situations sont évaluées: des puces adultes n'ayant jamais pris de repas et des puces adultes gorgées suite à un premier repas de sang.

Pour chacune des températures, 125 imagos de C. f. strongylus, de quatre jours d'âge, n'ayant jamais pris de repas sanguin, sont réparties dans cinq pots cylindriques $(3,5 \mathrm{~cm}$ de diamètre et $7 \mathrm{~cm}$ de haut) à raison de 25 puces par pot. En guise de support pour ces puces, un morceau de papier Whatman $\mathrm{N}^{\circ} 1$ de dimension $2,5 \times 6 \mathrm{~cm}$ plié en quatre est déposé à l'intérieur de chaque pot. À partir de la date du dépôt, chaque jour, les puces mortes sont retirées des pots par aspiration puis dénombrées.

Dans les mêmes conditions d'élevage que précédemment, 125 individus de C.f. strongylus, de deux jours d'âge et ayant passé deux autres jours à se gorger sur des chiennes de race Beagles sont collectés et répartis dans cinq pots cylindriques. À partir de la date du dépôt, chaque jour et pour chaque température, les puces mortes sont retirées des pots par aspiration puis dénombrées.

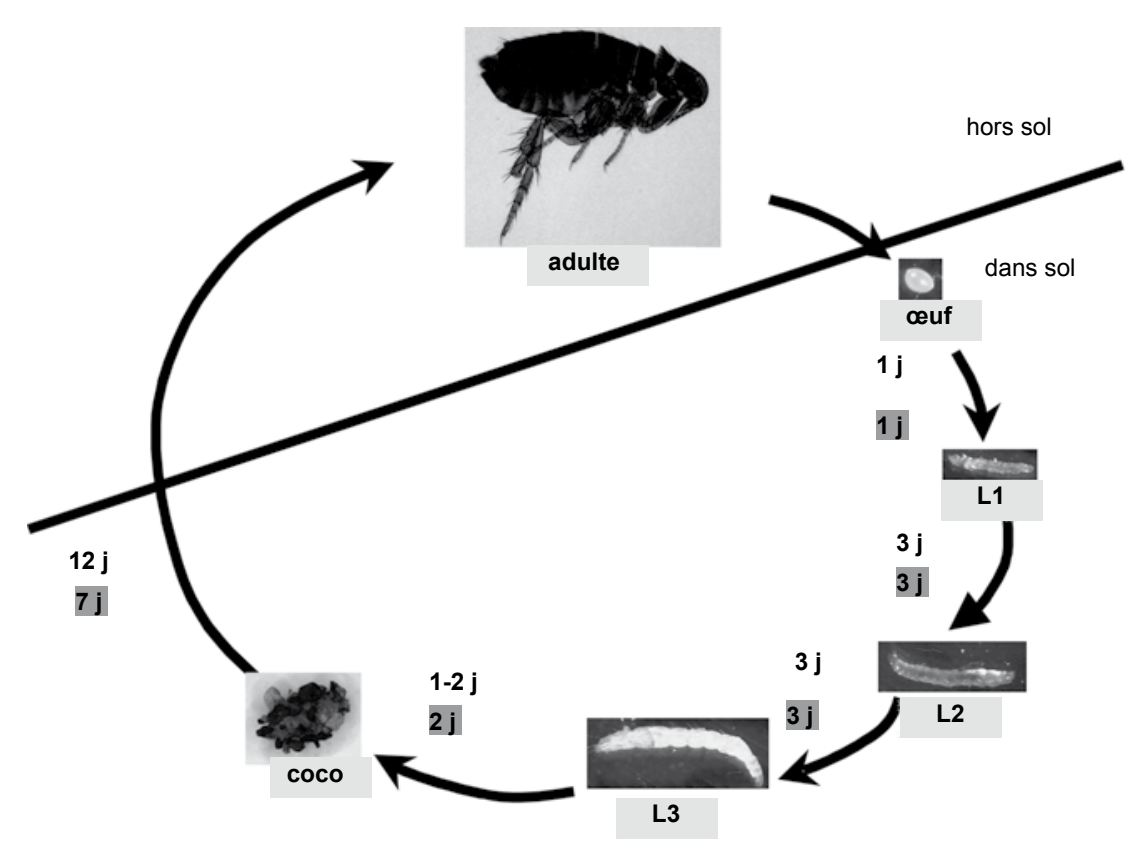

Fig. 1. - Durée minimale du cycle de développement de C.f. strongylus en fonction de la température.

$27^{\circ} \mathrm{C} \bowtie 21$ jours $29^{\circ} \mathrm{C} \bowtie 17$ jours 


\section{RÉSULTATS ET DISCUSSION}

\section{CyCle de DÉveloppement DE C. F. STRONGylus}

Le cycle évolutif de C.f. strongylus est relativement court à $29^{\circ} \mathrm{C}$ (16-17 jours), en comparaison avec celui obtenu à $27^{\circ} \mathrm{C}$ (20-21 jours) (Figure 1$)$. Il ressort de cette figure qu'à $27^{\circ} \mathrm{C}$ comme à $29^{\circ} \mathrm{C}$, le temps mis pour observer les premières L1 de C.f. strongylus est de un jour après la ponte, quatre jours après la ponte pour les L2, sept jours après la ponte pour les L3 et huit à neuf jours après la ponte pour les cocons. L'unique différence observée au cours du déroulement du cycle de C.f. strongylus, provient de la durée de nymphose qui est plus long à $27^{\circ} \mathrm{C}$ (12 jours) qu'à $29^{\circ} \mathrm{C}$ ( 7 jours). En effet, selon Dryden \& Gillard (1995), ce stade de développement est celui qui accroît la longévité de la puce dont la maturation est retardée par la baisse de la température.

En comparaison avec C.f. felis, il semblerait que le cycle de C.f. strongylus soit plus long pour des températures d'incubation identiques. Metzger \& Rust (1996) et Cadiergues (2000) ont effectivement obtenu, pour C.f. felis et à $27^{\circ} \mathrm{C}$, une durée du cycle évolutif de 15 jours, significativement plus courte que celle enregistrée à la même température avec C.f. strongylus. Cette différence au niveau de la durée du cycle de développement pourrait s'expliquer par l'effet des températures d'exposition des individus de chaque sousespèce, en rapport avec les températures qui règnent dans leurs aires de distribution respectives. En effet, Ménier \& Beaucournu (1999) ont montré que la présence de C.f. strongylus était liée à des climats beaucoup plus chauds (Afrique sub-saharienne) tandis que C.f. felis était plus présente dans les zones à climat tempéré (Afrique du Nord, Europe et Amérique).

SURVIE DE $C$. F. STRONGYLUS

\section{À DIFFÉRENTES TEMPÉRATURES}

Les taux de survie observés pour les individus à jeun, évoluent en fonction de la température (Figure 2). À $27^{\circ} \mathrm{C}$ et à $29^{\circ} \mathrm{C}$, ces taux sont sensiblement comparables. Après émergence et entre 27 et $29^{\circ} \mathrm{C}$, environ $50 \%$ des imagos à jeun de C.f. strongylus sont encore en vie, sept à huit jours après. Toutefois, 14 jours après émergence de ces mêmes imagos, aucun individu n'est retrouvé vivant dans le milieu. À $19^{\circ} \mathrm{C}$, par contre, les taux de survie observés de C.f. strongylus à jeun, présentent une évolution significativement distinctes des deux précédentes températures $(\mathrm{p}=0,0023)$. En définitive, aucune puce à jeun n'est observée dans le milieu environnant au-delà de 16 jours après émergence, à $19{ }^{\circ} \mathrm{C}$. Les résultats obtenus à partir de C.f. felis (Dryden, 1989 ; Silverman et al., 1991 ; Franc, 1998) indiquent que la durée de vie des individus à jeun des deux sousespèces de $C$. felis est semblable, quelle que soit la température. À $27^{\circ} \mathrm{C}$ par exemple, Silverman et al. (1991) ont montré que C.f. felis a un taux de survie de $10 \%$, six jours après l'émergence des adultes.

Après la prise du premier repas sanguin, les taux de survie de C.f. strongylus présentent toujours une évolution qui est fonction de la température (Figure 3). Contrairement à la situation précédente (puces à jeun), les taux de survie à chaque température se démarquent significativement les uns des autres $(p=0,0001)$. Moins de $50 \%$ des puces sont encore en vie, un jour après qu'elles soient libérées dans l'environnement à $29^{\circ} \mathrm{C}$. Par contre à 19 et $27^{\circ} \mathrm{C}$, on retrouve plus de $90 \%$ des adultes gorgés dans le milieu, un jour après leur libération. Passé trois jours hors de leur hôte à $29^{\circ} \mathrm{C}$, cinq jours à $27^{\circ} \mathrm{C}$ et huit jours à $19^{\circ} \mathrm{C}$, aucune puce gorgée de sang n'est retrouvée vivante dans l'environnement. Les résultats de Hink et al. (1991) et Dryden (1993) relatifs à $C$. felis corroborent les nôtres pour les températures élevées, dans la mesure où, pour ces auteurs, la puce du chat ne survivrait pas plus de 48 à 96 heures si elle est privée d'hôte alors qu'elle a déjà effectué un séjour de cinq jours sur un chat. Lorsque la puce du chat a entamé sa reproduction après les premiers repas sanguins, il semblerait qu'elle devienne dépendante de ces apports constants de sang, ce qui la rend vulnérable lorsqu'elle en est privée (Dryden \& Gillard, 1995) ; nos

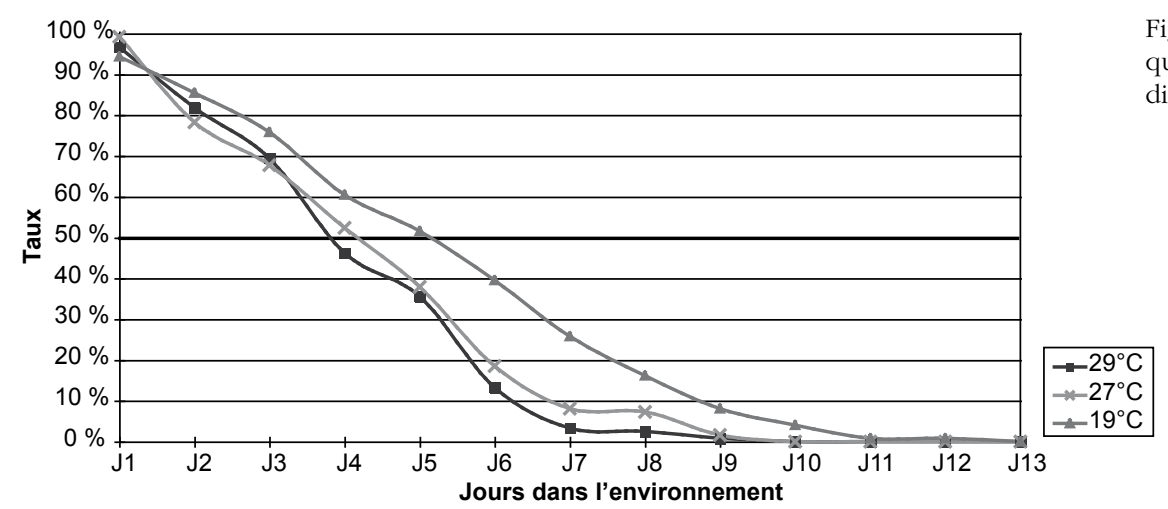

Fig. 2. - Taux de survie de C.f. strongylus de quatre jours d'âge, à jeun, en fonction de différentes températures. 


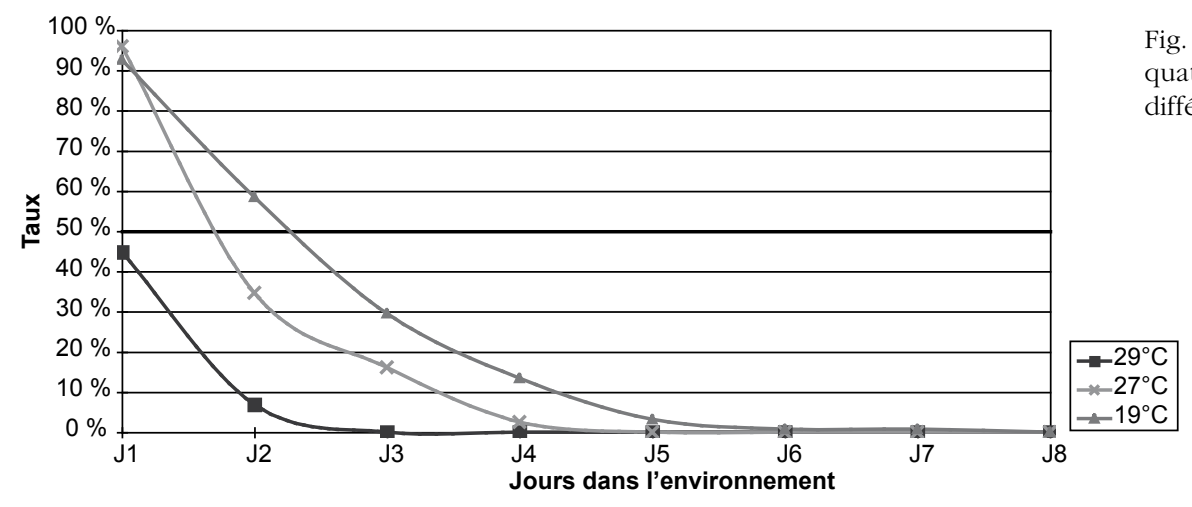

Fig. 3. - Taux de survie de C.f. strongylus de quatre jours d'âge, gorgée, en fonction de différentes températures.

résultats le confirment. Ainsi que présentée par Silverman \& Rust (1989) et Franc (1998) qui ont travaillé sur C. felis, probablement C.f.felis, la survie de la puce du chat dépendrait de son état de gorgement et des conditions ambiantes, comme la température et l'humidité relative.

\section{CONCLUSION}

$\tau$

es variations de température influencent certains paramètres bio-écologiques du cycle de développement de la puce du chat. L'étude de la durée du cycle évolutif de C.f. strongylus a montré qu'elle est plus longue que celle de C. f. felis pour une même température d'élevage. En effet, à $27^{\circ} \mathrm{C}$ par exemple, 21 jours sont nécessaires pour observer les premiers imagos de C.f. strongylus émerger ; contre 15 jours pour C.f. felis. En outre, les fortes températures réduisent la durée du cycle de développement de C.f. strongylus, soit 17 jours à $29^{\circ} \mathrm{C}$. Concernant le taux de survie des adultes de C.f. strongylus, il est également fonction de la température ambiante mais aussi de l'état de gorgement des individus. À jeun, C. $f$. strongylus autant que $C$.f. felis survivent relativement plus longtemps, environ 16 jours à $19^{\circ} \mathrm{C}$ et 14 jours à $27-29^{\circ} \mathrm{C}$. Lorsque les individus de ces deux sous-espèces ont pris leur premier repas de sang, on assiste à une diminution significative de leur durée de vie, environ huit jours à $19^{\circ} \mathrm{C}$, cinq jours à $27^{\circ} \mathrm{C}$ et trois jours à $29^{\circ} \mathrm{C}$.

Désormais, il est possible de prévoir, au vu des résultats disponibles, l'évolution des populations de C.f. strongylus suivant les modifications de températures. Afin de mener une lutte efficace contre cette sous-espèce de la puce du chat, il serait donc judicieux de tenir compte de ces informations actuellement disponibles, surtout que quelques différences ont été relevées au niveau de certains paramètres bio-écologiques des deux sous-espèces de C. felis, notamment le cycle de développement.

\section{REMERCIEMENTS}

Tous nos remerciements à Solange Vermot et Martine Roques pour leur aide active à ce travail. Remerciements également adressés au Directeur de l'ENVT, pour avoir accepté que de tels travaux soient entrepris dans l'institution qu'il dirige.

\section{RÉFÉRENCES}

CAdiergues M.C. Ctenocephalides canis (Curtis, 1826) (Siphonaptera : Pulicidae) : Données épidémiologiques et biologique. Thèse : Sciences Agronomiques, INP de Toulouse, 2000, 198 p.

Curasson G. Ctenocephalides canis, parasite du mouton. Bulletin de la société de Pathologie Exotique, 1925, 18, 755-756.

Dryden M.W. Biology of the cat flea, Ctenocephalides felis felis. Companion Animal Practice, 1989, 19 (3), 23-27.

Dryden M.W. Biology of fleas of dogs and cats. Compendium on Continuing Education for the Practising Veterinarian, 1993, 15 (4), 569-577.

Dryden M.W. \& Gillard R. Biologie de Ctenocephalides felis felis et lutte contre les puces du chien

ET du chat. Pratique médicale et chirurgicale de l'animal de compagnie, 1995, 30, 207-217.

Franc M. Ctenocephalides felis (Bouché, 1835) (Siphonaptera : Pulicidae) : Données épidémiologiques et biologiques, Méthodes d'évaluation des moyens de lutte. Thèse de Doctorat, Université Paul Sabatier, Toulouse III, 1998, 230 p.

Hink W.F., Drought D.C. \& BarnetT S. Effect of an experimental systemic compound, CGA-184699, on life stages of the cat flea (Siphonaptera: Pulicidae). Journal of Medical Entomology, 1991, 28 (3), 424-427.

KaAl J.F., Baker K. \& Torgerson P.R. Epidemiology of flea infestation of ruminants in Libya. Veterinary Parasitology, 2006, 141 (3-4), 313-318.

MéNIER K. \& Beaucournu J.C. Importance médico-vétérinaire des puces du genre Ctenocephalides Stiles \& Collins, 1930. Revue de Médecine Vétérinaire, 1999, 150 (8-9), 675-680. 
Metzger M.E. \& RusT M.K. Egg production and emergence of adult cat fleas (Siphonaptera: Pulicidae) exposed to different photoperiods. Journal of Medical Entomology, 1996, 33 (4), 651-655.

Opasina B.A. Ctenocephalides canis infestation of goats. Tropical Animal Health Production, 1983, 15, 106.

SÉGuY E. Ordre des Siphonaptères (Siphonaptera Latreille, 1798), in : Grassé P.P., Traité de Zoologie, AnatomieSystématique-Biologie, X, 1951, 745-769.

SILVERMAN J. \& RUST M.K. Extended longevity of the preemerged adult cat flea (Siphonaptera: Pulicidae) and factors stimulating emergence from the pupal cocoon. Annals of the Entomological Society of America, 1985, 78, 763-768.

Silverman J., Rust M.K. \& ReIERSON D.A. Influence of temperature and humidity on survival and development of the cat flea, Ctenocephalides felis (Siphonaptera: Pulicidae), Journal of Medical Entomology, 1991, 18 (1), 78-83.

YAO K.P. Situation des ectoparasitoses du chien domestique (Canis lupus familiaris Linné, 1759) (Canidae) à Abidjan : Cas spécifique de la pulicose à Ctenocephalides felis strongylus (Jordan, 1925) (Pulicidae). Thèse de Doctorat Unique de l'Université de Cocody/Abidjan, 2007, 210 p.

Reçu le 23 février 2010 Accepté le 10 mars 2010 